\title{
The Generalized Uncertainty Principle and Quantum Gravity Phenomenology
}

\author{
Ahmed Farag Ali, Saurya Das \\ Dept. of Physics, University of Lethbridge, 4401 University Drive, \\ Lethbridge, Alberta, Canada T1K 3M4 \\ E-mails:ahmed.ali@uleth.ca,saurya.das@uleth.ca \\ http://directory.uleth.ca/users/ahmed.ali, http://people.uleth.ca/ saurya.das \\ Elias C. Vagenas \\ Research Center for Astronomy \& Applied Mathematics, \\ Academy of Athens, \\ Soranou Efessiou 4, GR-11527, Athens, Greece \\ E-mail: evagenas@academyofathens.gr \\ http://users.uoa.gr/ evagenas
}

\begin{abstract}
In this article we examine a Generalized Uncertainty Principle which differs from the Heisenberg Uncertainty Principle by terms linear and quadratic in particle momenta, as proposed by the authors in an earlier paper. We show that this affects all Hamiltonians, and in particular those which describe low energy experiments. We discuss possible observational consequences. Further, we also show that this indicates that space may be discrete at the fundamental level.
\end{abstract}

Keywords: Quantum Gravity Phenomenology

String Theory, ${ }^{1}$ certain other approaches to Quantum Gravity, as well as Black Hole Physics ${ }^{2}$ suggest a modification of the Heisenberg's Uncertainty Principle near the Planck scale to a so-called Generalized Uncertainty Principle (GUP) of the form

$$
\Delta p \Delta x \geq \frac{\hbar}{2}\left[1+\beta_{0} \frac{\ell_{P l}^{2}}{\hbar^{2}} \Delta p^{2}\right]
$$

where $\ell_{P l}=\sqrt{\frac{G \hbar}{c^{3}}}=10^{-35} \mathrm{~m}$ is the Planck length and $\beta_{0}$ is a constant, normally assumed to be of order unity. Evidently, the new second term on the RHS of (1) is important only when $x, \Delta x \approx \ell_{P l}$ or $p, \Delta p \approx p_{P l} \approx 10^{16} \mathrm{TeV} / \mathrm{c}$ (the Planck momentum), i.e. at very high energies/small length scales. Inverting Eq.(1), we get $\Delta p \leq \frac{\hbar}{\beta_{0} \ell_{P l}^{2}}\left[\Delta x \pm \sqrt{\Delta x^{2}-\beta_{0} \ell_{P l}^{2}}\right]$, implying the existence of a minimum measurable length $\Delta x \geq \Delta x_{\min } \equiv \sqrt{\beta_{0}} \ell_{P l}$. It can be shown that the above GUP can be derived from a modified Heisenberg algebra ${ }^{3}$

$$
\left[x_{i}, p_{j}\right]=i \hbar\left[\delta_{i j}+\frac{\beta_{0} \ell_{P l}^{2}}{\hbar^{2}}\left(p^{2} \delta_{i j}+2 p_{i} p_{j}\right)\right] \text {. }
$$

On the other hand, Doubly Special Relativity (DSR) theories ${ }^{4}$ suggest yet another modified algebra between position and momenta ${ }^{5}$

$$
\left[x_{i}, p_{j}\right]=i \hbar\left[\left(1-\ell_{P l}|\vec{p}|\right) \delta_{i j}+\ell_{P l}^{2} p_{i} p_{j}\right]
$$

as well as the existence of a maximum observable momentum $\Delta p \leq \Delta p_{\max } \approx$ $M_{P l} c$. Using the Jacobi identity $\left[\left[x_{i}, x_{j}\right], p_{k}\right]+\left[\left[x_{j}, p_{k}\right], x_{i}\right]+\left[\left[p_{k}, x_{i}\right], x_{j}\right]=0$ and the 
assumption that space commutes with space and momenta with momenta, algebras (2) and (3) can be reconciled as limits of a single algebra of the form ${ }^{6} *$

$$
\left[x_{i}, p_{j}\right]=i \hbar\left[\delta_{i j}-\alpha\left(p \delta_{i j}+\frac{p_{i} p_{j}}{p}\right)+\alpha^{2}\left(p^{2} \delta_{i j}+3 p_{i} p_{j}\right)\right] .
$$

Here $\alpha=\frac{\alpha_{0}}{M_{P l} c}=\frac{\alpha_{0} \ell_{P l}}{\hbar}$. Again, $\alpha_{0}$ is normally assumed to be of order unity. The above algebra predict both a $\Delta x_{\min }$ and a $\Delta p_{\max }$. It also implies the following representation of the momentum operator in position space $p_{j}=p_{0 j}\left(1-\alpha p_{0}+2 \alpha^{2} p_{0}^{2}\right)$ where $p_{0 j}=-i \hbar \frac{\partial}{\partial x_{j}}$ is canonical (but unphysical) and satisfies the usual commutator $\left[x_{i}, p_{0 j}\right]=i \hbar \delta_{i j}$. Correspondingly, a non-relativistic Hamiltonian takes the form $H=\frac{p^{2}}{2 m}+V(\vec{r})=\frac{p_{0}^{2}}{2 m}+V(\vec{r})-\frac{i \hbar^{3} \alpha}{m} \frac{d^{3}}{d x^{3}}$ where the last term can be considered as a Quantum Gravity induced perturbation in the time-dependent Schrödinger Equation

$$
\left[H_{0}+H_{1}\right] \psi=\left[-\frac{\hbar^{2}}{2 m} \frac{d^{2}}{d x^{2}}+V(x)-i \frac{\alpha \hbar^{3}}{m} \frac{d^{3}}{d x^{3}}\right] \psi=i \hbar \frac{\partial \psi}{\partial t}
$$

The above equation admits of a new conserved current $J=\frac{\hbar}{2 m i}\left(\psi^{\star} \frac{d \psi}{d x}-\psi \frac{d \psi^{\star}}{d x}\right)+$ $\frac{\alpha \hbar^{2}}{m}\left(\frac{d^{2}|\psi|^{2}}{d x^{2}}-3 \frac{d \psi}{d x} \frac{d \psi^{\star}}{d x}\right)$ and charge $\rho=|\psi|^{2}$, such that $\frac{\partial J}{\partial x}+\frac{\partial \rho}{\partial t}=0$. The effect of the perturbation can be found for example on a simple harmonic oscillator, with $V=m \omega^{2} x^{2} / 2$, for which the shift in the ground state energy eigenvalues is, using second order perturbation theory $\frac{\Delta E_{G U P(0)}}{E_{0}} \sim \hbar \omega m \alpha^{2}$.

Concerning Landau Levels, for a particle of mass $m$, charge $e$ in a constant magnetic field $\vec{B}=B \hat{z} \approx 10 T, \vec{A}=B x \hat{y}$ and cyclotron frequency $\omega_{c}=e B / \mathrm{m}$, the Hamiltonian is $H=\frac{1}{2 m}\left(\vec{p}_{0}-e \vec{A}\right)^{2}-\frac{\alpha}{m}\left(\vec{p}_{0}-e \vec{A}\right)^{3}=H_{0}-\sqrt{8 m} \alpha H_{0}^{\frac{3}{2}}$ and the energy shifts are $\frac{\Delta E_{n(G U P)}}{E_{n}}=-\sqrt{8 m} \alpha\left(\hbar \omega_{c}\right)^{\frac{1}{2}}\left(n+\frac{1}{2}\right)^{\frac{1}{2}} \approx-10^{-27} \alpha_{0}$, from which we conclude that if $\alpha_{0} \sim 1$, then $\frac{\Delta E_{n(G U P)}}{E_{n}}$ is too small to measure. On the other hand, with current measurement accuracy of 1 in $10^{3}$, one obtains the following upper bound on the GUP parameter: $\alpha_{0}<10^{24}$.

Similarly for a Hydrogen atom with standard Hamiltonian $H_{0}=\frac{p_{0}^{2}}{2 m}-\frac{k}{r}$ and perturbing Hamiltonian $H_{1}=-\frac{\alpha}{m} p_{0}^{3}$, it can be shown that the GUP effect on the Lamb Shift is $\frac{\Delta E_{n(G U P)}}{\Delta E_{n}}=2 \frac{\Delta\left|\psi_{n l m(0)}\right|}{\psi_{n l m}(0)} \approx \alpha_{0} \frac{4.2 \times 10^{4} E_{0}}{27 M_{P l C^{2}}} \approx 10^{-24} \alpha_{0}$. Again, if $\alpha_{0} \sim 1$, then $\frac{\Delta E_{n(G U P)}}{E_{n}}$ is too small, whereas with current measurement accuracy of 1 in $10^{12}$, we infer $\alpha_{0}<10^{12}$. For some other examples, we refer the reader to our earlier papers ${ }^{7,8}$.

Finally, we consider the free-particle Schrödinger equation for a particle in a box of length $L,{ }^{6}$ with the solution $\psi(x)=A e^{i k^{\prime} x}+B e^{-i k^{\prime \prime} x}+C e^{\frac{i x}{2 \alpha \hbar}}$. Note the appearance of a new oscillatory term. Here $k^{\prime}=k(1+k \alpha \hbar), k^{\prime \prime}=k(1-k \alpha \hbar)$ (to leading order in $\alpha$ ). The boundary condition $\psi(0)=0$ implies $A+B+C=0$, and

*We also cite reference [6] for more references to earlier works. 
in addition to the boundary condition $\psi(L)=0$, this yields

$$
2 i A \sin (k L)=|C|\left[e^{-i\left(k L+\theta_{C}\right)}-e^{i\left(L / 2 \alpha \hbar-\theta_{C}\right)}\right]+\mathcal{O}\left(\alpha^{2}\right),
$$

where $C=|C| e^{-i \theta_{C}}$. Taking real parts of both sides (assuming $A$ is real, without loss of generality), we get $\cos \left(\frac{L}{2 \alpha \hbar}-\theta_{C}\right)=\cos \left(k L+\theta_{C}\right)=\cos \left(n \pi+\theta_{C}+\epsilon\right)$ which has the solutions

$$
\frac{L}{2 \alpha \hbar}=\frac{L}{2 \alpha_{0} \ell_{P l}}=n \pi+2 q \pi+2 \theta_{C} \text { or }=-n \pi+2 q \pi \quad[n, q \in \mathbb{N}] .
$$

From the above we conclude that a particle can be confined only in boxes of certain discrete lengths, and further speculate that this might indicate that all measurable lengths are quantized, since measurement of lengths require at least one particle, possibly many. We think that this result can be generalized to relativistic particles, as well as to the quantization of areas and volumes ${ }^{9}$.

In summary, in this article we have shown that a single GUP exists, which is consistent with the predictions of Black Hole Physics, String Theory, DSR etc., and that this induces perturbations to all Hamiltonians. Applying this to a few concrete examples such as the Harmonic Oscillator, Landau Levels and Lamb Shift, we have computed corrections due to this perturbation. From these, we concluded that if the GUP parameter $\alpha_{0}$ is of order unity, these corrections are probably too small to be measured at present. On the other hand, current experimental accuracies impose upper bounds on the GUP parameter. Finally, by solving the GUP corrected Schrödinger equation for a particle in a box, we have shown that boundary conditions require the box length to be quantized, suggesting quantization of measurable lengths, and possibly of surfaces and volumes as well. We hope to report further on these elsewhere.

This work is supported by the Natural Sciences and Engineering Research Council of Canada and the Perimeter Institute for Theoretical Physics.

\section{References}

1. D. Amati, M. Ciafaloni and G. Veneziano, Phys. Lett. B 216, 41 (1989).

2. M. Maggiore, Phys. Lett. B 304, 65 (1993) [arXiv:hep-th/9301067].

3. A. Kempf, G. Mangano and R. B. Mann, Phys. Rev. D 52, 1108 (1995) [arXiv:hepth/9412167].

4. G. Amelino-Camelia, Int. J. Mod. Phys. D 11, 35 (2002) [arXiv:gr-qc/0012051]; J. Magueijo and L. Smolin, Phys. Rev. Lett. 88, 190403 (2002) [arXiv:hep-th/0112090]. J. Magueijo and L. Smolin, Phys. Rev. D 71, 026010 (2005) [arXiv:hep-th/0401087].

5. J. L. Cortes and J. Gamboa, Phys. Rev. D 71, 065015 (2005) [arXiv:hep-th/0405285].

6. A. F. Ali, S. Das and E. C. Vagenas, Phys. Lett. B 678, 497 (2009) [arXiv:0906.5396 [hep-th]].

7. S. Das and E. C. Vagenas, Phys. Rev. Lett. 101, 221301 (2008) [arXiv:0810.5333 [hepth]].

8. S. Das and E. C. Vagenas, Can. J. Phys. 87, 233 (2009) [arXiv:0901.1768 [hep-th]].

9. A. F. Ali, S. Das, E. C. Vagenas, in preparation. 